\title{
Modification of Graphene and Friction and Wear Property of its Epoxy Resin Matrix Composites
}

\author{
Weisong Sun, Sirong Yu, Menglong Tang, Xian Wang \\ College of Mechanical and Electronic Engineering, China University of Petroleum, Qingdao266580, China.
}

\begin{abstract}
The graphene was modified by silane coupling agent KH560, the epoxy resin was reinforced by the modified graphene (KH-graphene) to produce KH-graphene/epoxy resin composites, and the effect of KH-graphene content and load on the friction and wear property of the composites was studied. The results showed that the KH560 was successfully grafted to the surface of graphene; The KH-graphene decreased the mass loss and friction coefficient of the epoxy resin, and with increasing the KHgraphene content, the mass loss and friction coefficient of KH-graphene/epoxy resin composites both showed a decreasing trend, and when the load was $150 \mathrm{~N}$, KH-graphene content was $0.5 \%$, the mass loss and friction coefficient of composites were reduced by $44.9 \%$ and $17.4 \%$; With increasing the load, the mass loss and friction coefficient of KH-graphene/epoxy resin composites also showed a decreasing trend; The wear form was mainly fatigue wear under the low load, and KH-graphene could inhibit the generation and expansion of micro-cracks; After the load increased, the wear form was mainly abrasive wear; After the graphene added, the wear scar of the worn surface of composites was relatively reduced.
\end{abstract}

Keywords: graphene; modification; epoxy resin; composites; friction and wear

In the field of machinery, how to reduce the friction caused by mechanical failure is still one of the biggest challenges. The researchers are trying to find perfect lubricants that reduce friction and wear without adding harmful chemicals or chemicals to the human body. The graphene has the perfect single-layer two-dimensional structure, its single carbon atoms are surrounded by three adjacent carbon atoms and in the form of SP2 hybrid followed by a positive hexagonal ${ }^{[1-3]}$. The special structure makes the graphene have excellent mechanical, thermal and electrical properties ${ }^{[4-6]}$. Shen ${ }^{[7]}$ studied the effect of graphene on the tribological properties of epoxy resin matrix. And the results show that the wear resistance of the epoxy resin was significantly enhanced when the graphene oxide was added, and the wear rate of the composite material was $90 \%$ lower than that of the pure epoxy resin when the graphene content was $0.5 \%$. Liu ${ }^{[8]}$ studied the sliding friction and wear behavior of graphene-based epoxy coatings in dry friction and seawater environments, respectively. The results show that the graphene can significantly reduce the friction coefficient and wear rate of epoxy coating in dry environment and seawater environment, and the friction coefficient and wear rate of graphene/epoxy coating in dry friction were lower than that in seawater environment.

In this paper, the surface modification of graphene was carried out with silane coupling agent KH560, which was served as the surface-active agent, and the changes of the structure and morphology of graphene were studied. The effects of the modified graphene (KH-graphene) content and the applied load on the friction and wear properties of KH-graphene/epoxy resin composites were studied.

\section{Experimental}

\subsection{Raw Materials}

The graphene (purity: $>95 \%$; thickness $0.55 \sim 3.74 \mathrm{~nm}$; plane size: $0.5 \sim 3 \mu \mathrm{m}$; layer: $1 \sim 10$ ) produced by Chengdu Organic Chemistry Co., Ltd. of Chinese Academy of Sciences was used as the reinforcing material. The silane coupling agent KH560 produced by Sinopharm Group Chemical Reagent Co., Ltd was used as the surfactant. The bisphenol A epoxy resin E51 produced by Nantong Star Synthetic Materials Co., Ltd was used as the matrix. The 2-ethyl-4-methylimidazole produced by Sinopharm Group Chemical Reagent Co., Ltd was used as the curing agent.

The silane coupling agent KH560 (whose molecular structure is shown in Figure.1) is the surface modification agent for the surface modification of carbon fibers, carbon nanotubes and other fillers to improve the compatibility of fillers in the epoxy resin [4]. The surface modification mechanism of graphene is shown in Figure.2. The surface modification process of graphene is as follows: (1) A certain amount of graphene and a certain amount of mixed acid (concentrated HNO3: concentrated H2SO4 = 3: 1) were mixed and stirring reaction for $6 \mathrm{~h}$; (2) After the mixture was cooled, the mixture was diluted with a quantity of deionized water and filtered repeatedly; (3) The product was dried in the freeze-drying oven for $24 \mathrm{~h}$ to obtain acidified graphene; (4) Then,

Copyright (C) 2020 Weisong Sun et al.

doi: $10.18282 / 1-$ e.v9i4.1657

This is an open-access article distributed under the terms of the Creative Commons Attribution Non-Commercial License (http://creativecommons.org/licenses/by-nc/4.0/), which permits unrestricted non-commercial use, distribution, and reproduction in any medium, provided the original work is properly cited. 
a certain amount of deionized water was measured and its $\mathrm{pH}$ value was adjusted to the $3.5 \sim 5.5$ by the hydrochloric acid; (5) A certain amount of KH560 was weighed and mixed with the deionized water to prepare the solution; (6) A certain amount of acidified graphene was added to the mixed solution and stirred for $6 \mathrm{~h}$; (7) After filtration, the product was placed in a freeze-drying oven for $24 \mathrm{~h}$ to obtain the KH-graphene.

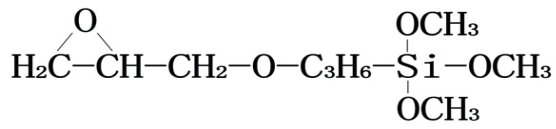

Figure.1 The molecular structure of silane coupling agent KH560

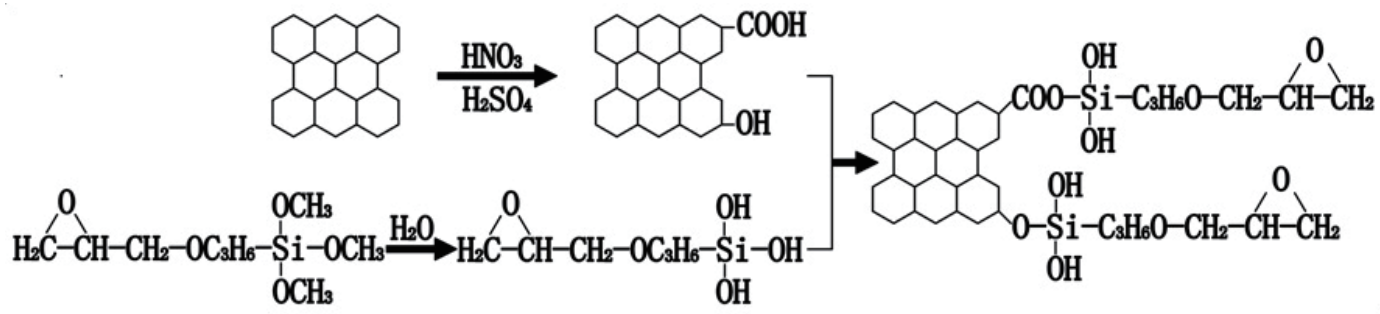

Figure.2 The surface modification mechanism of graphene

\subsection{Preparation of KH-graphene/epoxy resin composites}

The preparation process of the KH-graphene/epoxy resin composites is as follows: (1) A certain amount of KH-graphene was mixed with an appropriate amount of acetone; (2) The mixed system of KH-graphene and acetone was added to the epoxy resin and mechanically stirred for $3 \mathrm{~h}$; (3) The new mixed system was evacuated in the vacuum oven at $80{ }^{\circ} \mathrm{C}$ for $12 \mathrm{~h}$; (4) A solution of 2-ethyl-4-methylimidazole (3 wt.\% epoxy resin) was added to the mixture and stirred slightly for 10 min; (5) The mixture was vacuumed in a vacuum oven at $60{ }^{\circ} \mathrm{C}$ for $10 \mathrm{~min}$ and poured into the preheated mold; (6) The mixture was cured at $60{ }^{\circ} \mathrm{C}$ for $1 \mathrm{~h}$, cured at $80{ }^{\circ} \mathrm{C}$ for $1 \mathrm{~h}$, cured at $120^{\circ} \mathrm{C}$ for $5 \mathrm{~h}$, and sampled after cooling ${ }^{[9]}$.

\subsection{Characterization of properties}

The surface structures of graphene, acidified graphene and KH-graphene were analyzed by NEXUS infrared spectroscopy. The samples were mixed with $\mathrm{KBr}$ in an agate mortar for $15 \mathrm{~min}$ and pressed into a clear sheet, the resolution was $4 \mathrm{~cm}-1$, and the scanning range was $4000 \sim 400 \mathrm{~cm}-1$.

The friction and wear properties of pure epoxy resin and KH-graphene/epoxy resin composites were measured by M-2000A friction and wear tester. The samples were cut with 2000 \# sandpaper, and the sample size was $8 \times 10 \times 14 \mathrm{~mm}$. The material of the grinding ring was $45 \#$ steel, the hardness was $58.3 \mathrm{HRC}$, and the shape and size was shown in Figure.3. The test was dry sliding friction, the applied load was $50 \mathrm{~N}, 100 \mathrm{~N}$ and $150 \mathrm{~N}$, respectively, the wearing time was $60 \mathrm{~min}$, and the rotating speed of the grinding ring was $200 \mathrm{r} / \mathrm{min}$.
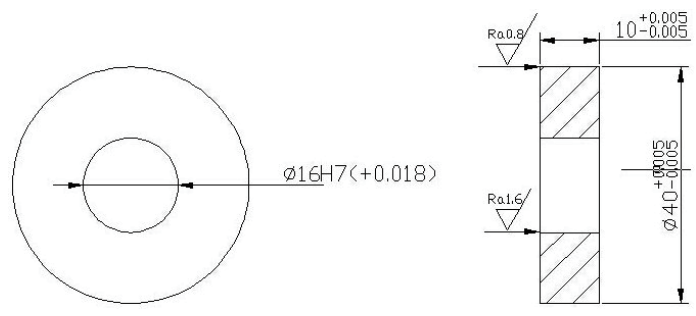

Figure.3 The shape and size of the grinding ring

The worn surfaces of graphene and KH-graphene and the micromorphologies of the worn surfaces of pure epoxy resin and KH-graphene/epoxy resin composites were observed by Nova Nano SEM 450 scanning electron microscope.

\section{Results and discussion}

\subsection{Analysis of surface modification of graphene}

Figure.4 shows the FT-IR plots of graphene, acidified graphene and KH-graphene. On the FT-IR curve of graphene, the absorption peak at $1561 \mathrm{~cm}-1$ corresponds to the $\mathrm{C}=\mathrm{C}$, and the absorption peak at $1217 \mathrm{~cm}-1$ corresponds to the C-C. On the FTIR curve of acidified graphene, the absorption peak at $3419 \mathrm{~cm}-1$ corresponds to the Hydroxyl(-OH), the absorption peak at 2359 $\mathrm{cm}-1$ corresponds to the $\mathrm{C} \equiv \mathrm{C}$ or $\mathrm{C}=\mathrm{C}=\mathrm{C}$, this indicates that acidification made the large $\pi$ delocalization of graphene destroyed ${ }^{[6]}$, the absorption peak at $1724 \mathrm{~cm}-1$ corresponds to the $\mathrm{C}=\mathrm{O}$ of carboxyl $(-\mathrm{COOH})$, and the absorption peak at $1400 \mathrm{~cm}-1 \mathrm{corresponds}$ to the $\mathrm{OH}$ of carboxyl (-COOH). In summary, the hydroxyl and carboxyl groups were successfully grafted onto the surface of graphene after the graphene was acidified by H2SO4 and HNO3. On the FT-IR curve of KH-graphene, the absorption peaks at $2921 \mathrm{~cm}-1$ and $2852 \mathrm{~cm}-1$ correspond to the anti-stretching vibration absorption peak and symmetrical stretching vibration absorption peak of $\mathrm{CH}$ of -CH2- of KH560, the absorption peaks at $1096 \mathrm{~cm}-1$ and $1041 \mathrm{~cm}-1$ correspond to the $\mathrm{Si}-\mathrm{O}-\mathrm{C}{ }^{[6,10]}$, and the absorption peak at $877 \mathrm{~cm}-1$ corresponds to the C-O-C ${ }^{[11,12]}$. In summary, the silane coupling agent KH560 was successfully grafted to the surface of graphene. 


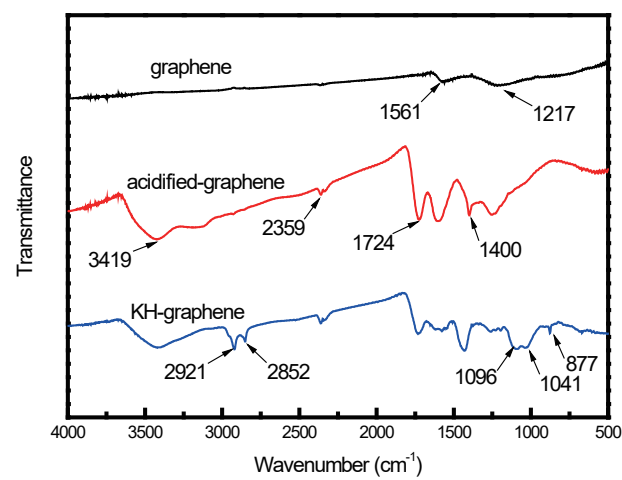

Figure.4 FT-IR spectrums of graphene, acidified graphene and KH-graphene

Figure.5 is the SEM image of graphene and KH-graphene. The graphene is layered and has a relatively smooth surface, but the sheet is dense and the dispersibility is poor. It can be seen that a large number of graphene are stacked together and form largesized graphene sheet. The surface of $\mathrm{KH}$-graphene is relatively rougher, the structure is looser, and the situation that the graphene is stacked together and forms large-sized graphene sheets is greatly reduced. The reason is that the coupling agent covered the surface of graphene and formed the smooth film after the surface modification. It made the interaction between the graphene layers greatly reduce, and reduced the agglomeration of graphene, so the $\mathrm{KH}$-graphene can be easy to disperse.

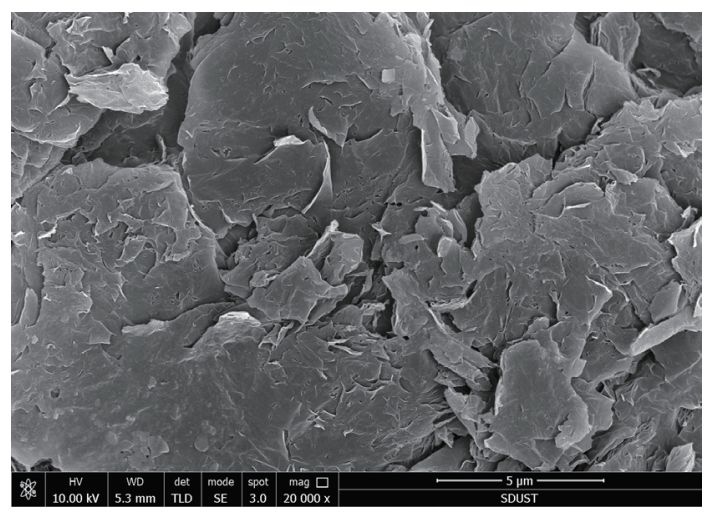

(a) graphene

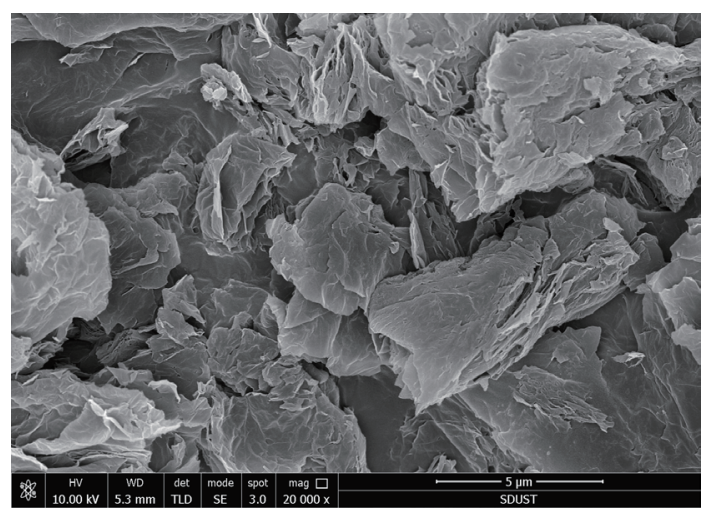

(b) KH-graphene

Figure.5 SEM pictures of graphene and KH-graphene

\subsection{Friction and wear properties of the composites}

\subsubsection{Effect of KH-graphene content on the friction and wear properties}

The variation rules of mass loss and friction coefficient of KH-graphene/epoxy resin composites at the applied load of $150 \mathrm{~N}$ are shown in Figure.6 and Figure.7, respectively. It can be seen from Figure.6 that the KH-graphene/epoxy resin composites has a smaller mass loss compared with the pure epoxy resin, and it indicates that the wear resistance of epoxy resin was improved after adding the appropriate amount of KH-graphene. With the increase of KH-graphene content, the mass loss of KH-graphene/epoxy resin composites decreased. When the $\mathrm{KH}$-graphene content was $0.5 \%$, the mass loss of $\mathrm{KH}$-graphene/epoxy resin composites decreased by $44.9 \%$ Compared with that of the pure epoxy resin. It can be seen from Figure.7 that the KH-graphene/epoxy resin composites had a smaller friction coefficient compared with the pure epoxy resin. With the increase of KH-graphene content, the friction coefficient of $\mathrm{KH}$-graphene/epoxy resin composites decreased. When the $\mathrm{KH}$-graphene content was $0.5 \%$, the friction coefficient of KH-graphene/epoxy resin composites decreased by $17.4 \%$ compared with that of the pure epoxy resin.

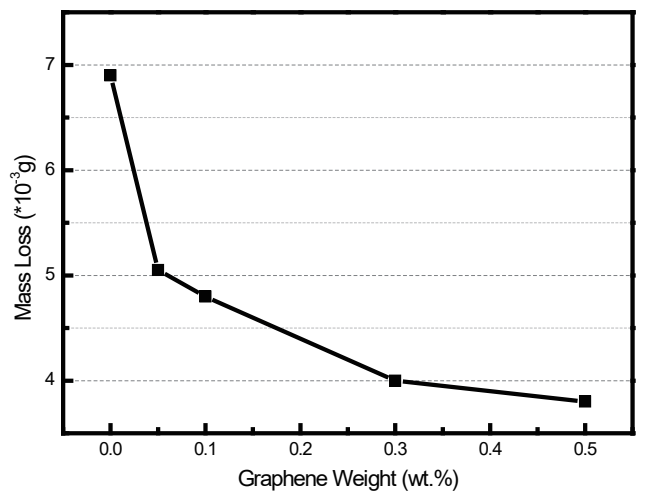

Figure.6 Mass losses of pure epoxy resin and KH-graphene/epoxy resin composites

at the applied load of $150 \mathrm{~N}$ 


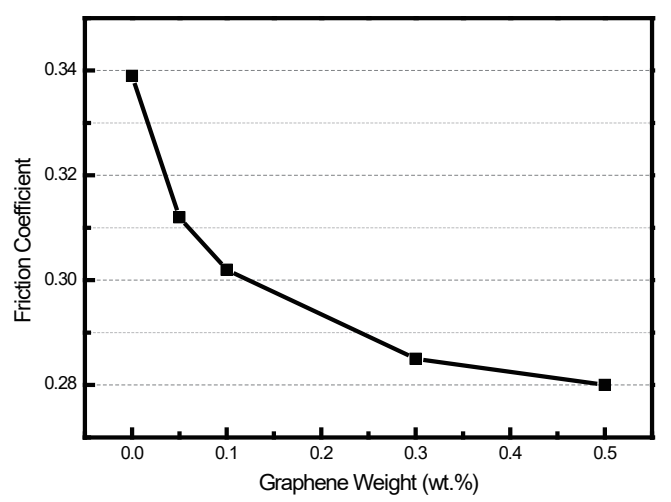

Figure.7 Friction coefficients of pure epoxy resin and KH-graphene/epoxy resin composites

at the applied load of $150 \mathrm{~N}$

The KH-graphene could improve the wear resistance of epoxy resin, the main reasons are as follows: (1) The KH-graphene dispersed in the matrix and the formed a network structure, which could improve the degree of cross-linking materials; (2) As a highly mechanically loaded sheet-like nanofiller, the KH-graphene had the effect of dispersion load, reducing stress concentration and pinning, and could alleviate the deformation and fragmentation of the matrix. And the larger surface area and the surface wrinkle shape and make KH-graphene have a good interface with the epoxy resin, which would make the separation of matrix particles difficult. So the bearing capacity of the composites was enhanced and the mass loss was reduced accordingly; (3) The $\mathrm{KH}$-graphene had the lubrication function. During the friction and wear process, the contact interface between the sample and the grinding ring formed a transfer film, which contained the KH-graphene. The KH-graphene made the transfer film have good stability and lubricity, so the transfer film could reduce the friction coefficient and mass loss.

\subsubsection{Effects of the applied load on the friction and wear properties}

The mass loss and friction coefficient of the KH-graphene/epoxy resin composites under different loads are shown in Figure.8 and Figure.9, respectively. It can be seen from Figure.8 that the mass loss of the KH-graphene/epoxy resin composites decreases with the increase of the applied load. When the KH-graphene content is $0.5 \%$, the mass loss of the composites is reduced by $60.0 \%$ as the load increases from $50 \mathrm{~N}$ to $100 \mathrm{~N}$. The mass loss of the composites decreased by $26.9 \%$ as the load increased from 100 $\mathrm{N}$ to $150 \mathrm{~N}$. It can be seen from Figure.9 that the friction coefficient of KH-graphene/epoxy resin composites decreases with the increase of the load. When the KH-graphene content is $0.5 \%$, the friction coefficient of the composites decreases by $30.3 \%$ with the increase of the load from $50 \mathrm{~N}$ to $100 \mathrm{~N}$. As the load increases from $100 \mathrm{~N}$ to $150 \mathrm{~N}$, the friction coefficient of the composites decreases by $18.1 \%$.

The reason is that the actual contact area between the specimen and the grinding ring is different under different loads, and the load affects the friction coefficient of the material by controlling the size of the actual contact area ${ }^{[9]}$. The material and the grinding ring is a flexible (or viscoelastic) contact state, when the load is small, the actual contact area is mainly dependent on the load and increases with increasing load. According to the adhesion theory, the friction force is proportional to the actual contact area, and the friction increases with the increase of the load, so the friction coefficient and load 1/3 times inversely proportional ${ }^{[13-14]}$, and the friction coefficient of the material decreases with increasing load. As the load is further increased, the contact state between the material and the grinding ring is changed from elastic (or viscoelastic) to plastic (or viscoplastic). The actual contact area mainly depends on the plastic deformation, the impact of the load on the friction coefficient weakened, so as the load increases the friction coefficient is still reduced but the decline becomes smaller and tends to be stable.

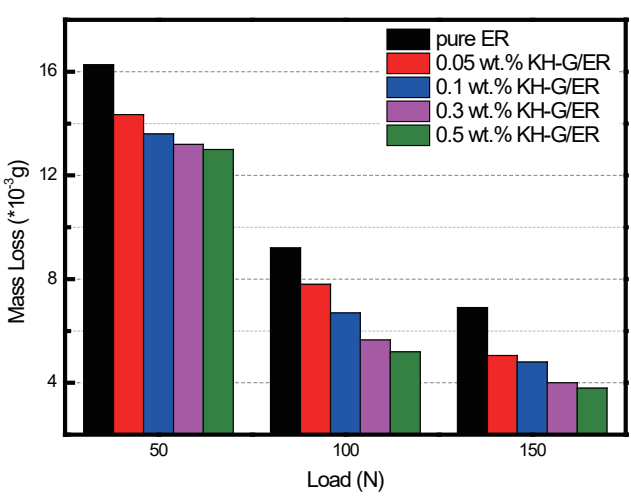

Figure.9 Mass losses of pure epoxy resin and KH-graphene/epoxy resin composites at different loads 


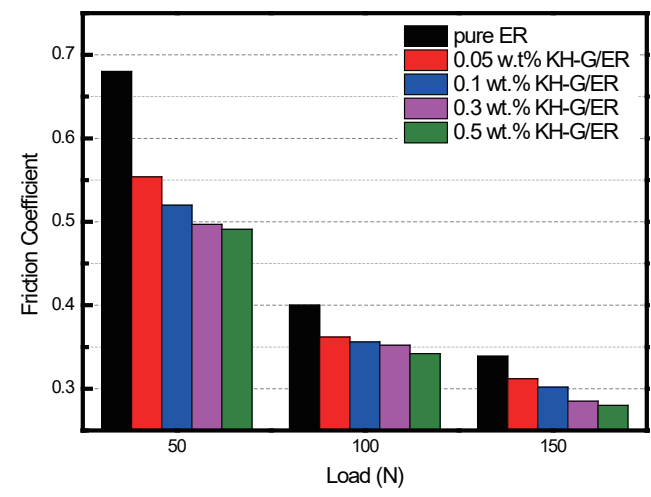

Figure.9 Friction coefficients of pure epoxy resin and KH-graphene/epoxy resin composites at different loads

\subsubsection{Wear mechanism of the composites}

In order to study the effect of KH-graphene content on the wear properties of epoxy resin, the worn surface morphologies of pure epoxy resin and the composites were observed. Figure.10 shows the worn surface morphology of pure epoxy resin and the composites under the applied load of $150 \mathrm{~N}$.

The worn surface of pure epoxy resin has a lot of furrows in Figure.10(a), and the wear is mainly abrasive wear. The reason is that in the friction and wear process the actual contact between the sample and the grinding ring is only a large number of microbumps on the sample surface. The epoxy resin is relatively soft, so the micro-bumps will deform, contact, adhere and produce micro-cracks under the action of the load. Under the action of the load, the micro-cracks will be further expanded and cause a portion of the microburst to fall off and form the debris from the specimen surface. With the wear and tear, the debris continued to accumulate at the contact interface between the sample and the grinding ring and produced the abrasive wear.

Compared with the worn surface morphology of pure epoxy resin in Figure.10(a), the furrow of the worn surface of the composite material in Figure.10(b) and Figure.10(c) were relatively reduced. The reason is as follows: (1) The graphene improved the load-carrying capacity of the epoxy resin matrix and inhibited the generation and expansion of the cracks, making it difficult to deform and tear the actual contact point with the grinding ring to form abrasive grains, resulting in better abrasion resistance; (2) During the friction and wear process, the transfer film containing graphene will be formed between the sample and the contact interface with the grinding ring, and the graphene makes the transfer film have good lubricity, so the worn surface is reduced.

The Figure.10(b) and Figure.10(c) show that the $0.5 \% \mathrm{KH}$-graphene/epoxy composites had less worn surface furrows. The reason is as follows: (1) The increase of graphene content makes the bearing capacity of the epoxy resin matrix further improved, so as to have better wear resistance; (2) When the graphene content is increased, the stability and lubricity of the transfer film will be improved.

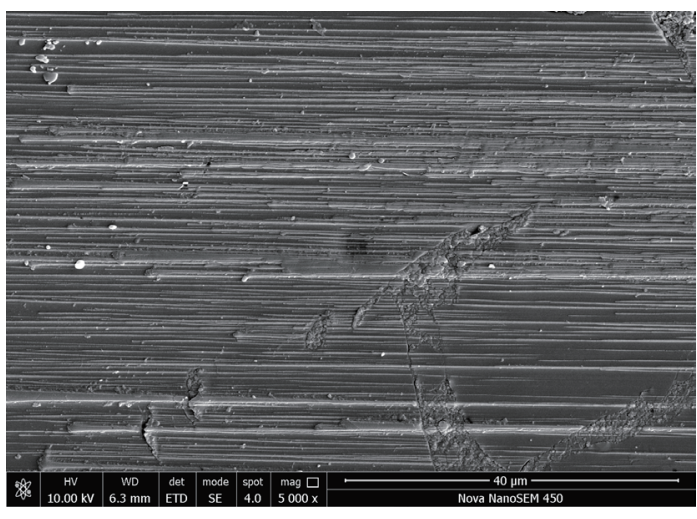

(a) $150 \mathrm{~N}$, pure ER

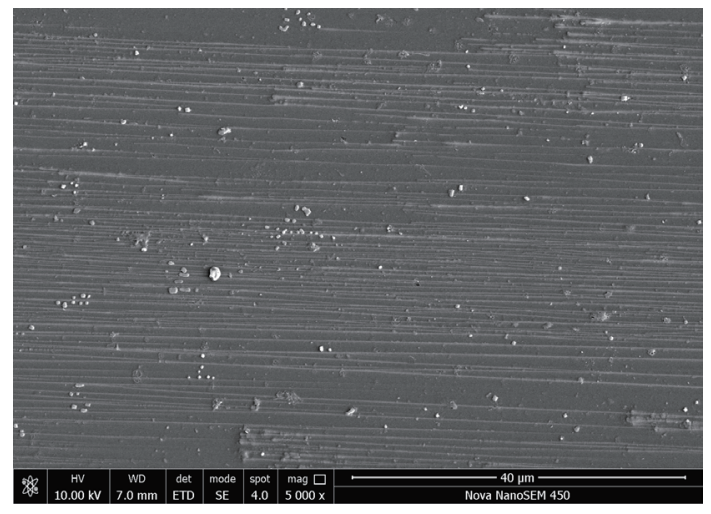

(b) $150 \mathrm{~N}, \quad 0.1 \% \mathrm{KH}$-graphene/ER 


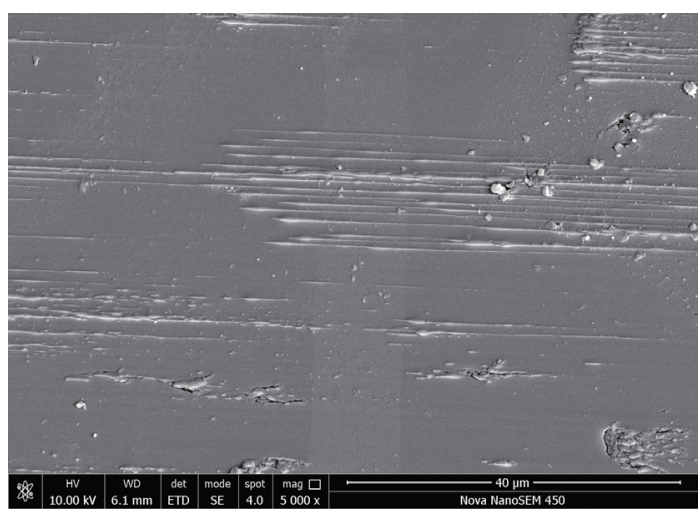

(c) $150 \mathrm{~N}, \quad 0.5 \% \mathrm{KH}$-graphene/ER

Figure.10 Worn surface features of pure epoxy resin, KH-graphene/epoxy resin

composites at the applied load of $150 \mathrm{~N}$

The worn surface morphologies of the pure epoxy resin and $\mathrm{KH}$-graphene/epoxy resin composites at $50 \mathrm{~N}$ and $100 \mathrm{~N}$ are shown in Figure.11, and the worn surface morphology at $150 \mathrm{~N}$ are shown in Figure.10 (a) and Figure.10 (c). In Figure.11 (a), the worn surface of the pure epoxy resin contains a large amount of microcracks when the load is $50 \mathrm{~N}$, and it exhibits typical fatigue wear. The reason is that the fatigue wear is one of the main wear forms of epoxy resin with harder wear on abrasive ring wear. The pure epoxy resin is relatively brittle, and the microvoids of the specimen surface will undergo micro-deformation and micro-melting under the cyclic loading. In this periodic deformation, due to the micro-pores, impurities and other defects of the sample surface and sub-surface, the sample will break in the molecular chain or intermolecular bond at the stress concentration of the surface and secondary surface, and it will produce frictional heat which can lead to the changes of the chain orientation and phase structure of the sample surface and secondary surface, so the microscopic tearing will occur at the sample surface and secondary surface. As the wears progress, the microcracks would gradually expand and cause some of the broken molecular segments to break away, resulting in fatigue wear ${ }^{[15]}$. Because the material and the grinding ring was the flexible (or viscoelastic) contact between the state at $\mathrm{r}$ low load. Compared with the gap between the sample and the grinding ring at high load, the gap was larger at low load. So the shedding is not easy to accumulate, in the contact surface of specimen and grinding ring, and the worn surface contains a large number of micro-cracks and almost no furrow at low load. In addition, since the radial load and the frictional heat are low at low load, so the interface of sample and grinding ring is not easy to form the transfer film. It failed to alleviate the grinding of the sample, so the wear is relatively large. Compared with pure epoxy resin, the worn surface of composites has less microcracks and the size of microcracks is smaller. The reason is that the graphene dispersed in the matrix and formed the network structure, which can improve the degree of cross-linking of materials and reduce stress concentration, making micro-cracks difficult to produce. In addition, since the graphene has the effect of suppressing the crack propagation, so even if a small amount of microcracks is generated, but they cannot be large-scale expansion due to the presence of graphene. This will control the size and quantity of microcracks in the material, slowing fatigue wear.

In Figure.11(c), there are some small furrows on the worn surface of the pure epoxy resin at $100 \mathrm{~N}$. The reason is that the micro-bumps of the specimen are in contact with the grinding ring. The epoxy resin is relatively soft, so the micro-bumps will be deformed, contact, adhesion and produce micro-cracks under the action of the load. As the wears progress, the microcracks will be further expanded, and a portion of the microburst will be destroyed and the debris will be formed. As the load increases, the contact state between the material and the grinding ring is changed from elastic (or viscoelastic) to plastic (or viscoplastic). The gap between the specimen and the grinding ring becomes smaller, so the abrasive is easy to accumulate between the specimen and the contact surface of the grinding ring, so there is a furrow on the worn surface. In addition, since the radial load and frictional heat are also increased due to the increase of the load, the transfer film which could slow the wear of the grinding ring on the specimen will form at the contact surface between the specimen and the grinding ring under the action of radial load and frictional heat. In contrast to Figure.11(c) and Figure.11(d), it was found that the surface roughing of the composites was relatively reduced as compared with the pure epoxy resin. The reasons are as follows: (1) The graphene improved the degree of crosslinking of the epoxy resin and reduced the stress concentration, so the micro-cracks were not easy to produce and can inhibit the propagation of cracks, so that the micro-bumps are not easily damaged to form the debris; (2) The graphene improved the carrying capacity of the epoxy resin, so the micro-bumps was not easy to deform, tear and form the debris, so the composites had better wear resistance; (3) During the friction and wear process, the transfer film containing the graphene would be formed at the contact interface between the sample and the grinding ring, the graphene would make the transfer film have good stability and lubricity. As a result, the furrows on the worn surface were reduced.

In Figure.10(a), there are a large number of the furrows on the worn surface of the pure epoxy resin at $150 \mathrm{~N}$, and the wear was mainly the abrasive wear. The reason is that the gap between the sample and the grinding ring was further reduced when the load was further increased, and the debris would accumulate, so there are a lot of furrows on the worn surface. As can be seen from Figure.10(a) and Figure.10(c), compared with the pure epoxy resin, the furrows of the composites were significantly reduced. The reason is that the radial load and frictional heat were further increased after the load was further increased. It would be easier to form the transfer films containing graphene, and the lubrication of graphene would be easier to play. 


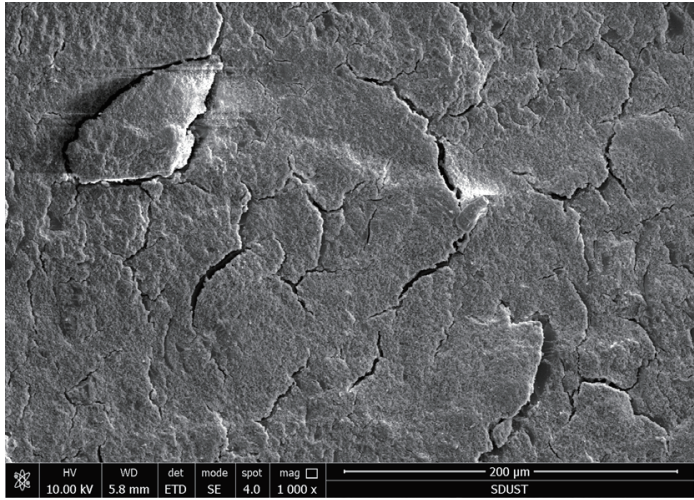

(a) $50 \mathrm{~N}$, pure ER

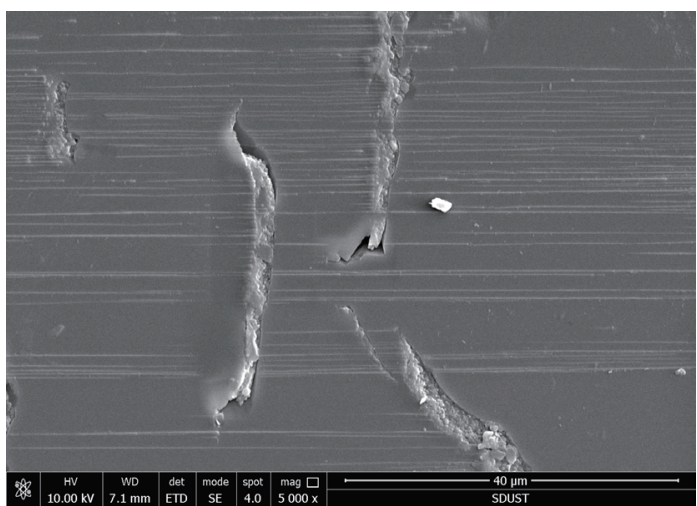

(c) $100 \mathrm{~N}$, pure ER

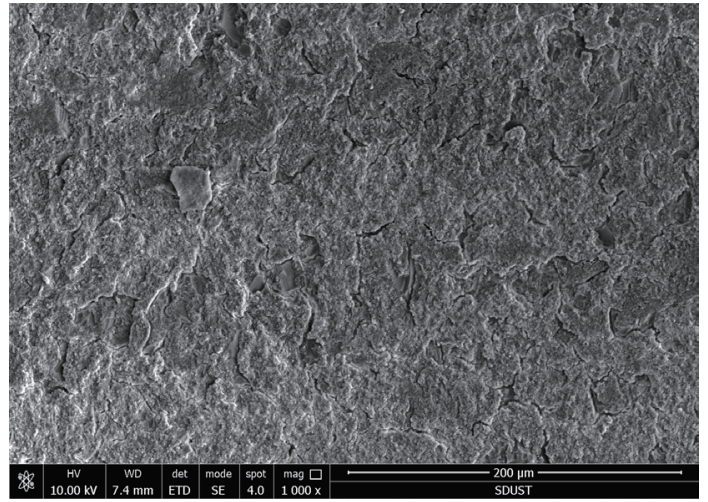

(b) $50 \mathrm{~N}, \quad 0.5 \% \mathrm{KH}$-graphene/ER

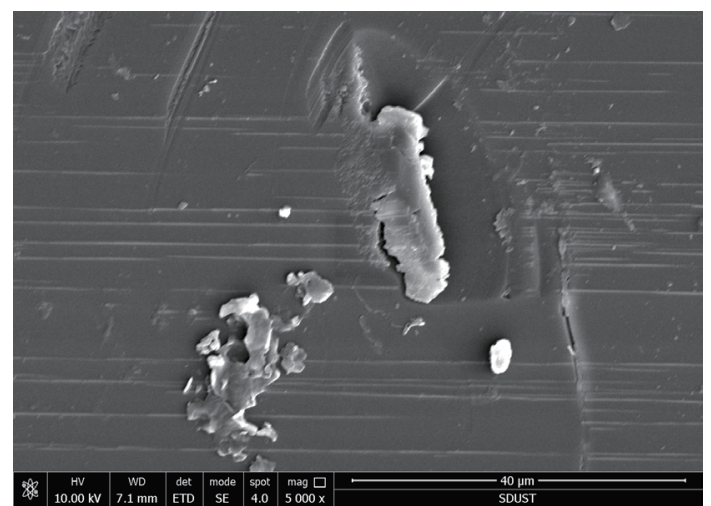

(d) $100 \mathrm{~N}, \quad 0.5 \% \mathrm{KH}$-graphene/ER

Figure.11 Worn surface features of pure ER, 0.5\% KH-graphene/ER composites at different loads

\section{Conclusions}

(1) The KH560 was successfully grafted onto the surface of graphene, and the KH-graphene/epoxy resin composites were prepared by using KH-graphene as reinforcement.

(2) After the addition of KH-graphene, the wear and friction coefficient of epoxy resin were reduced. With the increase of $\mathrm{KH}$-graphene content, the wear and friction coefficient of KH-graphene/epoxy resin composites decreased. When the load was $150 \mathrm{~N}$ and the $\mathrm{KH}$-graphene content was $0.5 \%$, the wear and friction coefficient of the composites were respectively reduced by $44.9 \%$ and $17.4 \%$.

(3) With the increase of load, the friction and friction coefficient of the KH-graphene/epoxy resin composites decreased. When the content of $\mathrm{KH}$-graphene was $0.5 \%$, the wear and friction coefficient of composites were reduced by $60.0 \%$ and $30.3 \%$, respectively, with the load increasing from $50 \mathrm{~N}$ to $100 \mathrm{~N}$. As the load increased from $100 \mathrm{~N}$ to $150 \mathrm{~N}$, the wear and friction coefficient of the composites decreased by $26.9 \%$ and $18.1 \%$, respectively.

(4) The wear mechanisms of pure epoxy resin and KH-graphene/epoxy resin composites under low load were mainly the fatigue wear, and the KH-graphene could inhibit the generation and expansion of micro-cracks. After the increase of the load, the wear mechanisms of pure epoxy resin and composites were mainly the abrasive wear, and the furrows of the composites were relatively few.

\section{References}

[1] Artur M. Pinto, Ines C. Goncalves, Fernao D. Magalhaes. Graphene-based materials biocompatibility: A review [J]. Colloids and Surfaces B: Biointerfaces, 2013, 111:188-202.

[2] Aidan O’Neill, Dimitri Bakirtzisb, Dorian Dixon. Polyamide 6/Graphene composites: The effect of in situpolymerisation on the structure and properties of graphene oxide and reduced graphene oxide [J]. European Polymer Journal, 2014, 59:353-362.

[3] Lu Peng, Feng Yiyu, Zhang Xuequan, et al. Recent progresses in application of functionalized graphene sheets [J]. Science China, 2010, 53(9):2311-2319.

[4] Guo W, Chen G. Fabrication of graphene/epoxy resin composites with much enhanced thermal conductivity via ball milling technique [J]. Journal of Applied Polymer Science, 2014, 131(15): 40565.

[5] Zaman I, Kuan H C, Dai J, et al. From carbon nanotubes and silicate layers to graphene platelets for polymer nanocomposites [J]. Nanoscale, 2012, 4(15): 4578-4586.

[6] Tapas Kuilla, Sambhu Bhadra, Dahu Yao, et al. Recent advances in graphene based polymer composites [J]. Progress in Polymer Science, 2010, 35:1350-1375.

[7] Shen Xiaojun, Pei Xianqiang, Fu Shaoyun, Klaus Friedrich. Significantly modified tribological performance of epoxy nanocomposites at very low graphene oxide content [J]. Polymer, 2013, 54: 1234-1242. 


\title{
Feasibility analysis of yoga on sports fatigue recovery of Competitive Aerobics
}

\author{
Yu Tan, ChengQing Tan \\ College of physical education, Hunan Normal University, Changsha, Hunan 410012 ,China.
}

\begin{abstract}
: competitive aerobics is a group of difficult and beautiful sports with high intensity, high difficulty and remarkable competitiveness. With the strengthening of sports training and competition, sports fatigue occurs from time to time. Some researchers have proved that yoga has a positive effect on the recovery of exercise-induced fatigue through implementation, Through the method of literature, this paper finds that yoga practice is of great significance to the recovery training of sports fatigue, so it puts forward that yoga practice can be applied to the recovery training of sports fatigue in competitive aerobics.
\end{abstract}

Keywords: Yoga; Competitive aerobics; Fatigue recovery

Yoga is a soft, low-intensity exercise, which has a positive effect on the physiological and psychological functions of the human body. The intensity of competitive aerobics is high and the technical difficulty is high. With the development of events, the number of events increases, the training and competition tasks become heavier, and the athletes' sports fatigue is more significant. which are the research on the causes, diagnosis methods and recovery means of sports fatigue of competitive aerobics, and lack of in-depth research on specific methods of fatigue recovery. It is unprecedented to apply yoga practice to the sports fatigue recovery training of competitive aerobics, so this paper puts forward the application of yoga to the sports fatigue recovery of competitive aerobics, and explores its feasibility and practical significance.

\section{1 .related concepts}

\subsection{Competitive Aerobics}

According to the International Gymnastics Federation aerobics competition rules, competitive aerobics is defined as a complete set of movements with continuous, complex and high-intensity characteristics under the accompaniment of music. It is a kind of sports with human health, strength, beauty, difficulty and overall quality ${ }^{[1]}$ 。

\subsection{Yoga}

The Sanskrit of yoga is yuj, which means connection and combination. Yoga,By following the order of body perception, people's body, mind and spirit can develop harmoniously, which is a health care method for people to practice and exercise ${ }^{[2]}$. Yoga is a philosophy, which can recognize oneself by exercising one's body, spirit and consciousness.

Copyright $(\mathcal{C} 2020$ Yu Tan et al.

doi: $10.18282 / 1-$-e.v9i4.1658

This is an open-access article distributed under the terms of the Creative Commons Attribution Non-Commercial License (http://creativecommons.org/licenses/by-nc/4.0/), which permits unrestricted non-commercial use, distribution, and reproduction in any medium, provided the original work is properly cited.

[8] Liu Shuan, Jiang Xin, Zhao Hai-chao, et al. Corrosion Resistance and Wear Property of Graphene-Epoxy Coatings [J]. Tribology, 2015, 35(5): 598-605.

[9] Hu Haixia, Yu Sirong, Ma Jun, Wang Yuhui. Surface chemical effects epoxy dry friction process [J] Tribology, 2007, 27 (3): 241-245.

[10] Wang Yong-kai. Research on preparation and properties of graphene/epoxy resin composites [D]. Zhengzhou: Zhengzhou University, 2013.

[11] CHEN Jian-jian. The interfacial propertie's study of epoxy resin/carbon fiber composite grafted graphene [D]. Wuxi: Jiangnan University, 2012.

[12] Christina A, Hacker, Kelly A, Anderson, Lee J. Comparison of Si-O-C interfacial bonding of alcohols and aldehydes on $\mathrm{Si}(111)$ formed from dilute solution with ultraviolet irradiation[J]. Langmuir 2005, 21: 882-889.

[13] WANG Xin. Functionalization of graphene and its epoxy composites, and study on their flame retardant properties and mechanism [D]. Beijing: University of Science and Technology of China, 2013.

[14] Tanaka K. Friction and wear of semicrystalline polymers sliding against steel under water lubrication [J]. Journal of Lubrication Technology, Transactions ASME, 1980, 102(4): 526-533.

[15] Shi G, Zhang M Q, Rong M Z, et al. Friction and wear of low nanometer Si3N4 filled epoxy composites [J]. Wear, 2003, 254 : 784-796. 